OPEN ACCESS

Edited by:

Daniel Ortuño-Sahagún

Universidad de Guadalajara, Mexico

Reviewed by:

Mark A. McCormick, University of New Mexico,

United States

QueeLim Ch'ng,

King's College London,

United Kingdom

${ }^{*}$ Correspondence:

Javier Alvarez

jalvarez@ibgm.uva.es

Received: 24 May 2018

Accepted: 31 December 2018

Published: 17 January 2019

Citation:

García-Casas P, Arias-del-Val J,

Alvarez-Illera $P$, Woinicz A,

de los Ríos C, Fonteriz RI, Montero M

and Alvarez J (2019) The

Neuroprotector Benzothiazepine

CGP37157 Extends Lifespan in

C. elegans Worms.

Front. Aging Neurosci. 10:440.

doi: 10.3389/fnagi.2018.00440

\title{
The Neuroprotector Benzothiazepine CGP37157 Extends Lifespan in C. elegans Worms
}

\begin{abstract}
Paloma García-Casas ${ }^{1}$, Jessica Arias-del-Val ${ }^{1}$, Pilar Alvarez-Illera ${ }^{1}$, Aneta Wojnicz ${ }^{2}$, Cristobal de los Ríos ${ }^{2}$, Rosalba I. Fonteriz ${ }^{1}$, Mayte Montero ${ }^{1}$ and Javier Alvarez ${ }^{1 *}$

${ }^{1}$ Department of Biochemistry and Molecular Biology and Physiology, Faculty of Medicine, Institute of Biology and Molecular Genetics (IBGM), University of Valladolid and CSIC, Valladolid, Spain, ${ }^{2}$ Department of Clinical Pharmacology, Instituto Teófilo Hernando, Instituto de Investigación Sanitaria la Princesa (IP), Hospital Universitario de la Princesa, Universidad Autónoma de Madrid (UAM), Madrid, Spain
\end{abstract}

The benzothiazepine CGP37157 has shown neuroprotective effects in several in vitro models of excitotoxicity involving dysregulation of intracellular $\mathrm{Ca}^{2+}$ homeostasis. Although its mechanism of neuroprotection is unclear, it is probably related with some of its effects on $\mathrm{Ca}^{2+}$ homeostasis. CGP37157 is a well-known inhibitor of the mitochondrial $\mathrm{Na}^{+} / \mathrm{Ca}^{2+}$ exchanger (mNCX). However, it is not very specific and also blocks several other $\mathrm{Ca}^{2+}$ channels and transporters, including voltagegated $\mathrm{Ca}^{2+}$ channels, plasma membrane $\mathrm{Na}^{+} / \mathrm{Ca}^{2+}$ exchanger and the $\mathrm{Ca}^{2+}$ homeostasis modulator 1 channel (CALHM1). In the present work, we have studied if CGP37157 could also induce changes in life expectancy. We now report that CGP37157 extends C. elegans lifespan by 10\%-15\% with a bell-shaped concentrationresponse, with high concentrations producing no effect. The effect was even larger (25\% increase in life expectancy) in worms fed with heat-inactivated bacteria. The worm CGP37157 concentration producing maximum effect was measured by high-performance liquid chromatography-tandem mass spectrometry (HPLC-MS/MS) and was close to the $\mathrm{IC}_{50}$ for inhibition of the $\mathrm{Na}^{+} / \mathrm{Ca}^{2+}$ exchanger. CGP37157 also extended the lifespan in eat-2 mutants (a model for caloric restriction), suggesting that caloric restriction is not involved in the mechanism of lifespan extension. Actually, CGP37157 produced no effect in mutants of the TOR pathway (daf15/unc24) or the insulin/insulin-like growth factor-1 (IGF-1) pathway (daf-2), indicating that the effect involves these pathways. Moreover, CGP37157 was also ineffective in nuo-6 mutants, which have a defect in the mitochondrial respiratory chain complex I. Since it has been described that neuroprotection by this compound in cell cultures is abolished by mitochondrial inhibitors, this suggests that life extension in C. elegans and neuroprotection in cell cultures may share a similar mechanism involving mitochondria.

Keywords: C. elegans, CGP37157, lifespan, aging, neuroprotection, $\mathrm{Ca}^{2+}$ signaling, mitochondria, $\mathrm{Na}^{+} / \mathrm{Ca}^{2+}$ exchanger 


\section{INTRODUCTION}

The benzothiazepine CGP37157 has been shown to act as a neuroprotectant in several experimental models of neurotoxicity. CGP37157 rescued neuronal death induced by veratridine in both chromaffin cells and rat hippocampal slices, with an $\mathrm{EC}_{50}$ of 5-10 $\mu \mathrm{M}$ (Nicolau et al., 2009, 2010). It also protected rat hippocampal slices against glutamate or ischemia/reperfusionelicited stress (González-Lafuente et al., 2012) and SH-SY5Y human neuroblastoma cells subjected to $70 \mathrm{mM} \mathrm{K}^{+}$stimulation (Martínez-Sanz et al., 2015). It has also been shown that CGP37157 protects primary cultures of rat cortical neurons during NMDA insults, probably through inhibition of voltagedependent $\mathrm{Ca}^{2+}$ channels (VDCCs; Ruiz et al., 2014). However, it is remarkable that CGP37157 did not protect either chromaffin cells or rat hippocampal slices against the combination of the mitochondrial oxidative phosphorylation inhibitors oligomycin A + rotenone (Nicolau et al., 2010; González-Lafuente et al., 2012).

CGP37157 has been used for many years as a selective inhibitor of the mitochondrial $\mathrm{Na}^{+} / \mathrm{Ca}^{2+}$ exchanger (mNCX), the main mitochondrial $\mathrm{Ca}^{2+}$ efflux pathway. Nevertheless, a series of off-target effects of CGP37157 has been described. They include inhibition of L-type VDCC (Baron and Thayer, 1997), plasma membrane $\mathrm{Na}^{+} / \mathrm{Ca}^{2+}$ exchangers (Czyz and Kiedrowski, 2003), or $\mathrm{Ca}^{2+}$ homeostasis modulator 1 (CALHM1) $\mathrm{Ca}^{2+}$ channels (Moreno-Ortega et al., 2015). Many of these additional effects occur in the same range of concentrations. Therefore, it is difficult to attribute the effects of the drug to a particular molecular effect. Regarding the neuroprotective effect, it could be due to a combination of the effects CGP37157 has on different $\mathrm{Ca}^{2+}$ flux pathways. It has been recently shown that a designed hybrid compound of CGP37157 and the L-type $\mathrm{Ca}^{2+}$ channel inhibitor nimodipine had larger neuroprotective activity than any of the compounds separately when tested on in vitro cellular and tissue slices models related to cerebral ischemia (Buendia et al., 2017).

Many neuroprotective drugs have anti-aging activity as well (Cooper et al., 2015; Zárate et al., 2017). In the case of CGP37157, most of its known targets are also present in $C$. elegans, a well-known model for lifespan studies, and therefore a similar type of effects should be expected. Regarding the $\mathrm{mNCX}$, in fact, the diversity of isoforms is much higher in C. elegans. Humans have only one isoform of the mNCX, named NCLX (Palty et al., 2010). Instead, C. elegans has 10 different isoforms (named $n c x-1$ to $n c x-10$ ) and their functional role is not known in detail (Sharma et al., 2013; He and O'Halloran, 2014; Sharma and O'Halloran, 2014). One of them, $n c x-9$, has been reported to perform CGP37157-sensitive $\mathrm{Na}^{+} / \mathrm{Ca}^{2+}$ exchange activity in mitochondria (Sharma et al., 2017). C. elegans has also a single CALHM1 homolog, named clhm-1 (Tanis et al., 2013). It is present in the plasma membrane of muscle cells and sensory neurons and works as well as a VDCC regulated by extracellular $\mathrm{Ca}^{2+}$. Knock-out of CALHM1 produces altered locomotion and its overexpression is toxic, producing degeneration through a $\mathrm{Ca}^{2+}$-dependent mechanism (Tanis et al., 2013). As to L-type $\mathrm{Ca}^{2+}$ channels, C. elegans has only one gene encoding an L-type $\alpha 1$ VDCC subunit, named egl-19 and responsible for the action potentials in pharynx and body wall muscle (Lee et al., 1997; Jospin et al., 2002; Shtonda and Avery, 2005; Gao and Zhen, 2011; Liu et al., 2011), but the effect of CGP37157 on the egl-19 $\mathrm{Ca}^{2+}$ channel has not been tested.

In the present work, we have studied the effect of CGP37157 on lifespan in C. elegans nematodes. Our data show that submaximal CGP37157 concentrations extended lifespan in wild-type worms and in eat-2 mutants, a model of caloric restriction. The increase in life expectancy was even larger in worms fed with heat-inactivated bacteria. Instead, CGP37157 had no effect in mutants of two well-known nutrientsensitive pathways: daf-15/unc-24 (TOR signaling pathway) and daf-2 insulin-like growth factor-1 (IGF-1 signaling pathway). CGP37157 also had no effect on nuo-6 mitochondrial respiratory chain mutants. This reminds the lack of neuroprotective effect of CGP37157 on the neuronal death induced by mitochondrial respiratory chain inhibitors (Nicolau et al., 2010; GonzálezLafuente et al., 2012), and suggests that lifespan extension and neuroprotection induced by CGP37157 may occur by a similar mechanism involving mitochondria.

\section{MATERIALS AND METHODS}

\section{C. elegans Strains and Maintenance}

Strains used were as follows: AQ2038, an integrated strain expressing cytosolic cameleon 2.1. (YC2.1) in pharynx controlled by the myo-2 promoter (pmyo-2::YC2.1; Alvarez-Illera et al., 2016), kindly provided by Drs. Robyn Branicky and W. Schafer, MRC Laboratory of Molecular Biology, Cambridge, UK. Used here as a control. Its lifespan was not significantly different from that of the N2 strain (data not shown). Mutant eat-2(ad1113), nuo-6(qm200), daf-15(m81)/unc-24(e138), unc-24(e138) and daf2(e1370) strains were obtained from the Caenorhabditis Genetics Center. Heterozygotes unc-24(e138)/+ were obtained by crossing the unc-24(e138) strain with the AQ2038 strain. Worms were maintained and handled as previously described (Stiernagle, 2006). NGM agar plates were seeded with Escherichia coli (OP50). Strains were maintained at $20^{\circ} \mathrm{C}$.

\section{Administration of CGP37157 to the Worms}

CGP37157 is a very lipophilic drug, showing very poor water solubility (Pei et al., 2003; Martínez-Sanz et al., 2016). This property may difficult the accessibility and distribution of the drug in the worms. Thus, we have not only assayed the effect of CGP37157 dissolved in the NGM agar, but we have also used inclusion compounds with $\gamma$-cyclodextrin as a vehicle for drug administration. The $\gamma$-cyclodextrin inclusion compounds were prepared as described before (Kashima et al., 2012). Briefly, a $230 \mathrm{mg} / \mathrm{ml}$ water solution of $\gamma$-cyclodextrin was mixed 10:1 with a $50 \mathrm{mM}$ DMSO solution of CGP37157 or with a $25 \mathrm{mM}$ EtOH solution of cholesterol, stirred in the shaker at $1,200 \mathrm{rpm}$ during $20 \mathrm{~h}$ and centrifuged at $12,500 \mathrm{rpm}$ for $10 \mathrm{~min}$. The supernatant was carefully discarded and the resulting inclusion compound was dried in the hood, weighed 
and dissolved in M9 buffer. The inclusion compounds in the amounts indicated were mixed with OP50 for addition to the plates.

\section{C. elegans Lifespan Assays}

Eggs were obtained as described previously (Stiernagle, 2006) and transferred to E. coli (OP50) seeded NGM plates, either control plates or plates prepared in the presence of the required drug. For each assay, around 100 synchronized young adults (day 1 ) were transferred to E. coli (OP50) seeded NGM plates $(35 \mathrm{~mm}$ plates, 10 worms/plate) containing $15 \mu \mathrm{M}$ Fluorodeoxyuridine (FUdR) to avoid progeny. Control and drug-containing assays were always carried out in parallel. Plates were scored for dead worms every day. Worms that did not respond to touch with a platinum wire were considered dead. Age refers to days following adulthood. Plates with fungal contamination during the first 10 days of the assay were excluded from the study. Missing worms, individuals with extruded gonad or desiccated by crawling in the edge of the plate were censored, as well as plates with fungal contamination after the first 10 days. Control and drug-containing plates were kept close together in a temperature-controlled incubator set at $20^{\circ} \mathrm{C}$. Statistical analysis was performed with the SPSS software, using the Kaplan-Meier estimator and the log-rank routine for significance.

For the experiments with dead bacteria, OP50 were grown overnight at $37^{\circ} \mathrm{C}$ and then heat inactivated at $65^{\circ} \mathrm{C}$ for $30 \mathrm{~min}$. After treatment, an aliquot was found not to grow when placed in LB medium. NGM plates were then seeded with heat-killed bacteria and the rest of the assay was as described above.

\section{C. elegans Fertility Measurement}

Freshly OP50 seeded NGM plates were prepared without FUdR and with or without $50 \mu \mathrm{M}$ CGP37157. Then, 1 L4 larva was transferred to each plate and the number of eggs laid was counted every $24 \mathrm{~h}$ after transferring the worm to a new plate.

\section{Measurement of CGP37157 in C. elegans Worms}

Around 4,000 C. elegans worms were transferred at day 1 of adulthood to treatment plates containing $1 \mu \mathrm{g}$ of CGP37157containing $\gamma$-cyclodextrin inclusion compound mixed with OP50. At day 8 of treatment, worms were collected and washed by centrifugation at 2,000 rpm with cold water. The supernatant was removed and $500 \mu \mathrm{L}$ of cold methanol was added. Worms were resuspended and sonicated during 50 cycles (5 s on/5 s off). The suspension obtained was centrifuged at 5,000 rpm for $5 \mathrm{~min}$. The supernatant was collected and stored at $-80^{\circ} \mathrm{C}$ until its analysis by high-performance liquid chromatography-tandem mass spectrometry (HPLC-MS/MS).

\section{Determination of CGP37157 From C. elegans Extracts by HPLC-MS/MS}

CGP37157 quantification was performed using a HPLC-MS/MS system. The instrument consisted of HPLC, 1200 Series separation module (Agilent Technologies, Santa Clara, CA, USA) coupled to triple quadrupole mass spectrometer (MS/MS, 6410 series) equipped with electrospray ionization source
(ESI). HPLC-MS/MS system was controlled by Agilent Mass Hunter Workstation Data Acquisition software. The MS/MS was operating in positive multiple reaction monitoring mode and the conditions were set as followed: desolvation gas $\left(\mathrm{N}_{2}\right)$ flow $12 \mathrm{~L} / \mathrm{min}$, nebulizer pressure $60 \mathrm{psi}$, drying gas temperature $300^{\circ} \mathrm{C}$ and capillary voltage $4,000 \mathrm{~V}$. The $\mathrm{m} / \mathrm{z}$ ratios for the CGP37157 quantifier and qualifier ions were 324.1 > 214.1 and $324.1>179.1$, respectively. The HPLC separation was carried out at $25^{\circ} \mathrm{C}$ in a reversed-phase $\mathrm{C} 18$ column (ZORBAX Eclipse $\mathrm{XDB}, 4.6 \mathrm{~mm} \times 150 \mathrm{~mm}$ and $5 \mu \mathrm{m}$ particle size; Agilent Technologies, Santa Clara, CA, USA) protected by a $0.2-\mu \mathrm{m}$ on-line filter. $0.2 \%$ formic acid in water, $\mathrm{pH}=3.0(\mathrm{~A})$ and $0.2 \%$ formic acid in $\mathrm{ACN}(\mathrm{B} ; 30: 70, \mathrm{v} / \mathrm{v})$ were used as the mobile phase. The chromatogram was run under gradient conditions at a flow rate of $0.8 \mathrm{~mL} / \mathrm{min}$. The following gradient program was used for CGP37157 separation: $70 \%$ of B at $0.0-0.5 \mathrm{~min}$; gradually increasing phase $\mathrm{B}$ to $100 \%$ at $0.5-1.0 \mathrm{~min}$; $100 \%$ of $B$ at $1.0-2.0 \mathrm{~min}$; returning to the initial conditions (30\% of $\mathrm{A}$ and $70 \%$ of $\mathrm{B}$ ) at $2.0-2.5 \mathrm{~min}$; followed by a re-equilibration time of $2.5 \mathrm{~min}$, to give a total run time of $5 \mathrm{~min}$. Five $\mu \mathrm{L}$ of CGP37157 was injected into the chromatographic system.

\section{Materials}

CGP37157 was synthesized as previously described (MartínezSanz et al., 2015). $\gamma$-cyclodextrin was purchased from PanReac, Barcelona, Spain. FuDR was acquired from Alfa Aesar, Karlsruhe, Germany. Other reagents were obtained from Sigma, Madrid, Spain or Merck, Darmstadt, Germany.

\section{RESULTS AND DISCUSSION}

Because of the poor water solubility of CGP37157, we have used two different methods of drug administration to the nematodes. First, we used $\gamma$-cyclodextrin to generate an inclusion compound which is then added to the NGM plates together with the OP50. In this way, the drug is ingested by the worms together with the OP50 and directly absorbed into the intestine. Once prepared as described in "Materials and Methods," the inclusion compound is weighed and dissolved in M9 buffer, so that a known amount (in $\mu \mathrm{g}$ of inclusion compound) is added to the plates. The second method was to directly dissolve the compound in NGM agar at the maximum possible concentration, which was $25-150 \mu \mathrm{M}$.

Table 1 shows the results of a series of lifespan assays performed with several concentrations of CGP37157, added by any of the two methods to wild-type worms. Figure 1 shows plots of typical lifespan assays obtained for each condition. The plots correspond to the assays labeled in bold in Table $\mathbf{1}$. Figures $\mathbf{1 A - C}$ show the effect of three different amounts of the inclusion compound, $0.1,1$, and $3 \mu \mathrm{g}$. Figures $1 \mathrm{D}-\mathrm{F}$ show the effect of three concentrations of CGP37157 (50, 100 and 150 $\mu \mathrm{M})$ directly dissolved in NGM agar. Figure 1F summarizes the mean increases in lifespan obtained. In the case of the inclusion compound, the maximum lifespan extension was obtained at $1 \mu \mathrm{g}$, which increased lifespan by nearly $10 \%$. Concentrations below or above that level produced a much lower effect or even no effect. Direct effects of $\gamma$-cyclodextrin on 
TABLE 1 | Treatment of wild-type worms with CGP37157.

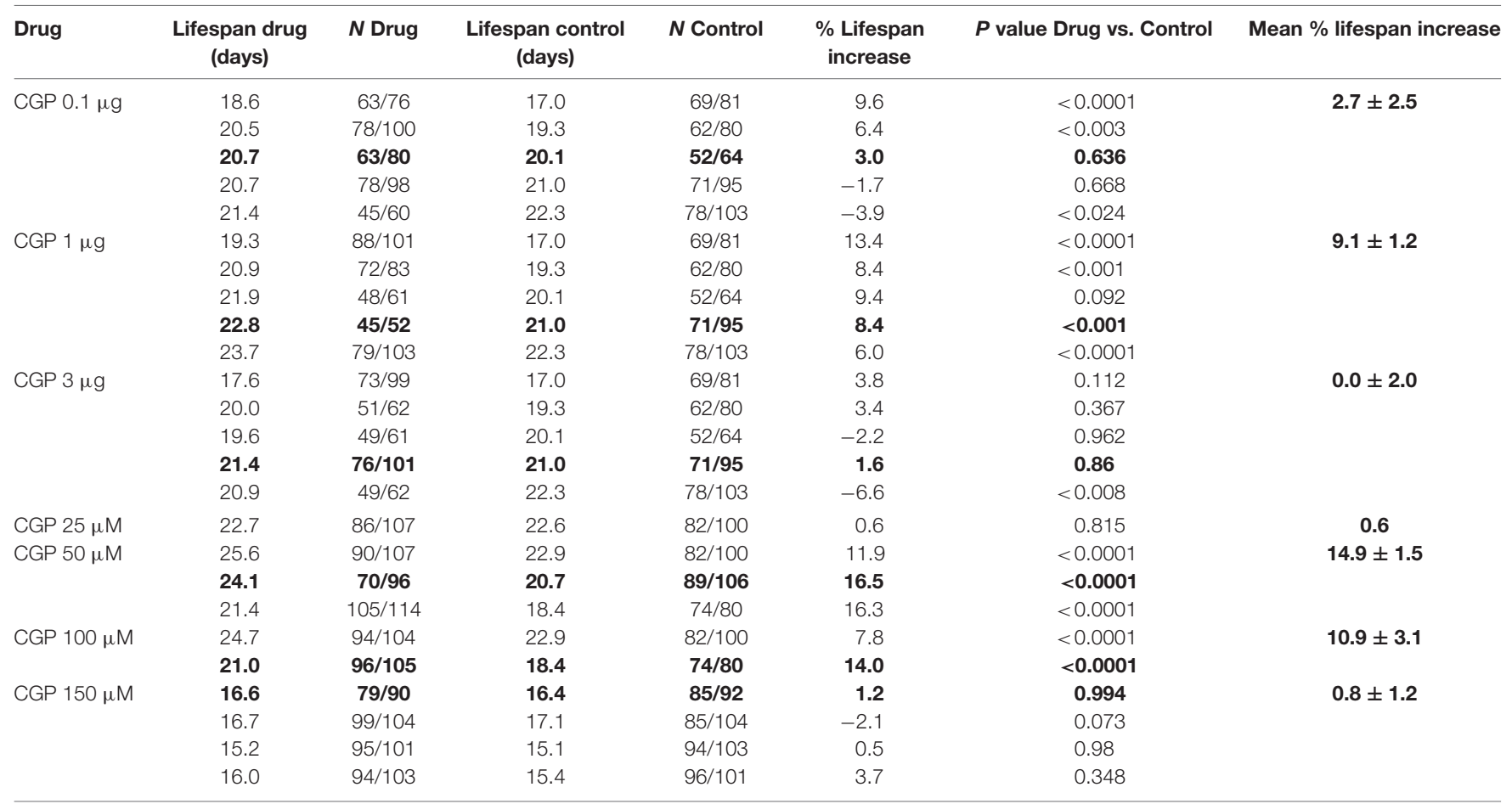

Lifespan assays performed with CGP37157 in wild-type worms. The table shows the drug concentration used in each series of assays, the half-life ( $T \frac{1}{2}$ ) of the worms incubated with the drug obtained from the Kaplan-Meier analysis, the number of worms in the drug-containing assay (final/total), the half-life ( $T \frac{1}{2}$ ) of the control worms, the number of worms in the control assay (final/total), the \% increase in the half-life, the statistical significance of the difference between control and treated worms, obtained from the log-rank test, and the mean $\pm S E$ increase in half-life from all the series made with the same drug concentration. Concentrations in $\mu g$ for $\gamma$-cyclodextrin-inclusion compounds and in $\mu M$ for compound dissolved in NGM agar. In bold, series shown in the survival plots of Figure 1.

C. elegans lifespan were excluded by studying the effect of a $\gamma$ cyclodextrin-cholesterol inclusion compound, which produced no effect (Table 2). Moreover, similar or even larger effects were obtained when CGP37157 was directly added to the NGM agar, showing that the effect was not dependent on the administration pathway. Both 50 and $100 \mu \mathrm{M}$ CGP37157 extended lifespan by $10 \%-15 \%$ with high statistical significance, but again here increasing the concentration to $150 \mu \mathrm{M}$ produced no effect. A lower concentration, $25 \mu \mathrm{M}$, was also ineffective (see Table 1). Therefore, CGP37157 is able to extend the C. elegans lifespan in a certain concentration range, suggesting that submaximal inhibition of one or more of their targets is required.

To exclude the possibility that the effect of CGP37157 could be the result of a secondary metabolite elicited by bacterial action on this compound, we have studied the effect of this compound on the lifespan of $C$. elegans worms fed with heat-inactivated OP50. The results are in Table 2. CGP37157 was still able to increase the $C$. elegans lifespan under these conditions. In fact, the effect was much larger, producing a mean increase in survival above $25 \%$. This suggests that bacterial metabolism of this compound could be interfering with its effect.

To investigate the mechanism of the lifespan extension induced by CGP37157, we have used several C. elegans mutants: eat-2(ad1113), nuo-6(qm200), daf-15(m81)/unc-24(e138), unc-24(e138) and daf-2(e1370). Mutant eat-2 has a defect in pharyngeal pumping that reduces the rate of feeding. This produces an increase in the survival of the mutant worms and is considered to be a model for the effects of caloric restriction (Lakowski and Hekimi, 1998). Therefore, if the effects of CGP37157 would be mediated by caloric restriction, we would expect it to produce little or no effect in eat-2 mutants. However, when we added the CGP37157 inclusion compound to eat-2 mutants, it produced the same effects than in wild-type worms, it increased by nearly $15 \%$ the lifespan at $1 \mu \mathrm{g}$, and produced no effect at $3 \mu \mathrm{g}$. Table 3 shows the results of a series of lifespan assays performed with these two CGP37157 concentrations in eat-2 mutants and panels A,B in Figure 2 show plots of typical assays performed at each concentration. The lifespan was longer in the eat-2 mutants than in the wild-type worms (compare with Table 1), and $1 \mu \mathrm{g}$ of CGP37157 increased it further by nearly $15 \%$. Instead, $3 \mu \mathrm{g}$ of the compound had no effect, similarly to wild-type worms. These data suggest that the lifespan extension induced by CGP37157 is not due to caloric restriction.

Then, we tested the effect of the maximally effective amount of CGP37157 inclusion compound, $1 \mu \mathrm{g}$, on nuo-6 mutants. These mutants have a defect in a subunit of complex I of the mitochondrial respiratory chain, and show reduced mitochondrial function, lower oxygen consumption, slow growth and movement (Yang and Hekimi, 2010b) and decreased ATP levels (Yee et al., 2014). This is accompanied by a significant lifespan extension, underscoring the importance of mitochondrial metabolism in survival (Yang and Hekimi, 2010b). In these mutants, however, we could not find any 


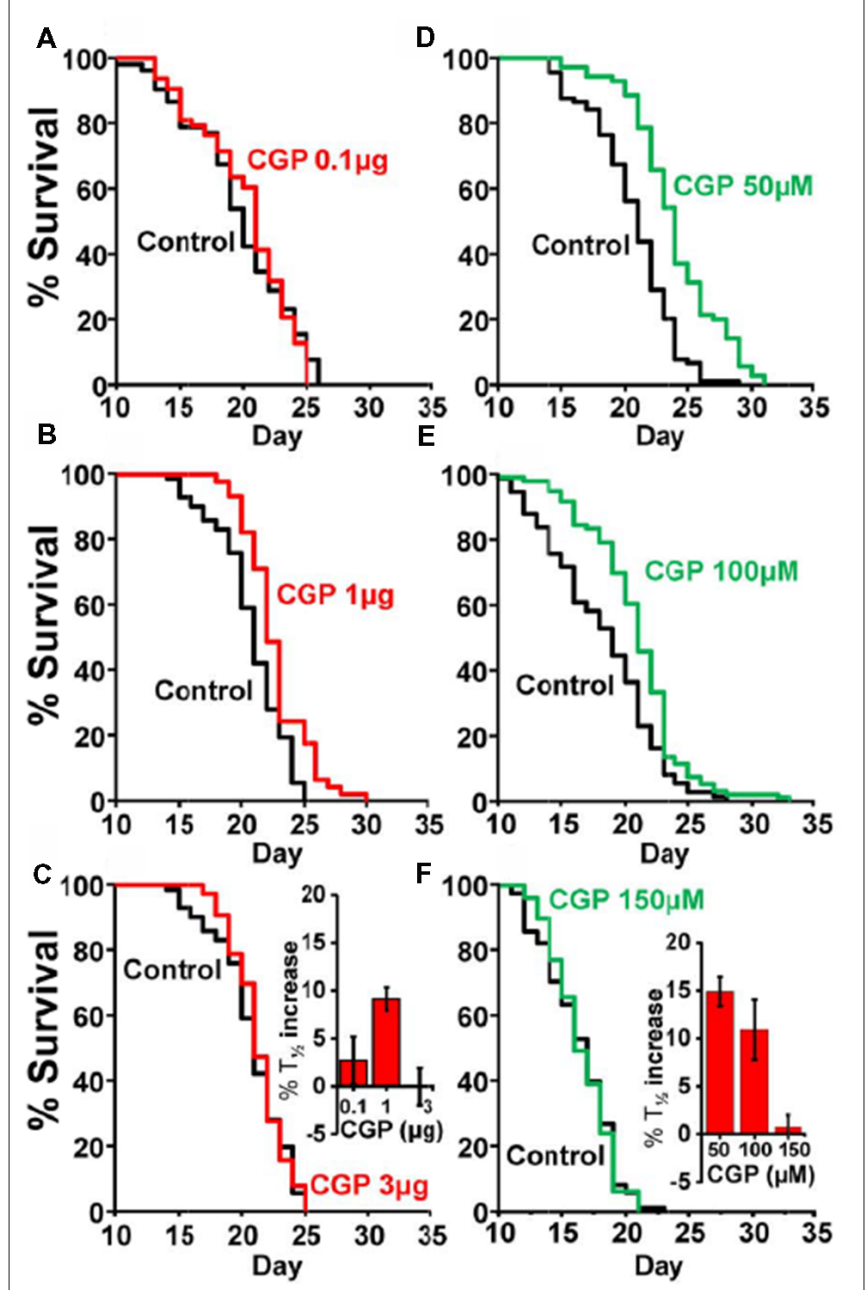

FIGURE 1 | Effects of CGP37157 on survival in C. elegans. Panels (A-C) show representative survival plots corresponding to parallel lifespan assays performed using $\gamma$-cyclodextrin-inclusion compounds in the following conditions: Control/CGP37157 (A: $0.1 \mu \mathrm{g}$; B: $1 \mu \mathrm{g}$; C: $3 \mu \mathrm{g}$ ). Panels (D-F) show representative survival plots from Control/CGP37157 lifespan assays in which either $50 \mu \mathrm{M}, 100 \mu \mathrm{M}$ or $150 \mu \mathrm{M}$ CGP37157 were dissolved in the NGM agar. The assays shown correspond to those marked in bold in Table 1. The insets placed in panels (C,F) show the mean increase in survival obtained in several similar lifespan assays of each kind (more details of all the assays in Table 1).

significant effect of GCP37157 on lifespan. Table 3 shows the results of a series of lifespan assays performed with $1 \mu \mathrm{g}$ of CGP37157 in nuo-6 mutants, and panel $\mathbf{C}$ in Figure 2 shows one of the lifespan assays. The lack of effect of CGP37157 in nuo-6 mutants reminds the lack of neuroprotective activity CGP37157 has in cellular models treated with mitochondrial oxidative phosphorylation inhibitors (Nicolau et al., 2010; González-Lafuente et al., 2012). Although the mechanism of the effects of CGP37157 in both cases is still unknown, it is clear that both effects require functional mitochondria to develop. In this respect, we should note that the only known mitochondrial target of CGP37157 is the mNCX. Therefore, this exchanger could play a role in these effects.
In the case of the daf-15/unc-24 mutants, as with nuo-6 mutants, we tested the effect of $50 \mu \mathrm{M}$ CGP37157 and we found no effect. The lack of effect was due to the DAF-15 mutation, because CGP37157 extended the lifespan of unc$24 /+$ mutants as much as in the controls (Table 3). daf-15 (raptor) heterozygous mutants have a partial suppression of the TOR pathway that produces an increase in lifespan of $13 \%$ (Jia et al., 2004). The lack of effect of CGP37157 in these mutants suggests that this pathway is somehow involved in the increase in life expectancy induced by this compound. Genetic and pharmacological inhibition of the TOR pathway has been shown to extend lifespan in many organisms, including C. elegans (Hansen et al., 2007). However, mitochondrial ROS-dependent TOR signaling has also been shown to be necessary for the lifespan extension induced by hypoxia (Schieber and Chandel, 2014). Given that mitochondrial $\mathrm{Ca}^{2+}$ is a critical regulator of ROS production (Görlach et al., 2015), changes in mitochondrial $\mathrm{Ca}^{2+}$ induced by CGP37157 could act on TOR signaling in this way. However, much further work is necessary to clarify this point.

Finally, our data also show that CGP37157 has little or no effect on daf-2 mutants. The daf-2 gene encodes for the insulinlike growth factor 1 (IGF-1) receptor, one of the best known nutrient-sensitive signaling pathways controlling lifespan (Gami and Wolkow, 2006). The lack of effect of CGP37157 in both daf$15 /$ unc-24 and daf-2 mutants is consistent with the overlap that exists between both the TOR and the insulin/IGF-1 signaling pathways. The expression of DAF-15 (raptor) is negatively regulated by DAF-16, a FOXO transcription factor that is in turn negatively regulated by daf- 2 insulin/IGF- 1 signaling. daf-15 (raptor) transcription is therefore regulated by daf-2 insulin/IGF signaling (Jia et al., 2004; Lapierre and Hansen, 2012). In addition, ROS have also been reported to be very important mediators for the increase in lifespan of daf-2 mutants (Zarse et al., 2012; Senchuk et al., 2018).

To estimate the long-term stability of CGP37157 in the assays, as well as the effective concentration attained by the drug inside the worms when it induces lifespan extension, we have determined the concentration of CGP37157 in the worms at day 8 of adult life during a typical lifespan assay with $1 \mu \mathrm{g}$ of the inclusion compound. Worm extracts were obtained twice, and CGP37157 was measured in triplicate samples of each extract. Considering a mean worm volume of $5 \mathrm{nl}$ (So et al., 2011), the concentration values obtained were $1.48,1.45$, and $1.57 \mu \mathrm{M}$ (extract 1) and 2.04, 2.18, and $2.16 \mu \mathrm{M}$ (extract 2). The mean value was $1.81 \pm 0.14 \mu \mathrm{M}$. This value is close to the $\mathrm{IC}_{50}$ for the inhibition of the mNCX by CGP37157 (Hernández-SanMiguel et al., 2006), although other targets of CGP37157 are also inhibited in the same concentration range. As mentioned in the Introduction, all the known targets of this compound participate in $\mathrm{Ca}^{2+}$ homeostasis, and it may seem reasonable to suggest a role for $\mathrm{Ca}^{2+}$ signaling in the mechanism of its effects. However, at this moment we cannot conclusively establish this point. In any case, the presence of CGP37157 in day 8 worms shows that the compound is stable under our experimental conditions and reaches concentrations in worms able to have a continuous submaximal inhibitory effect 
TABLE 2 | Control experiments: effect of $\gamma$-CD-cholesterol and effect of treatment with CGP37157 in wild-type worms fed with dead OP50.

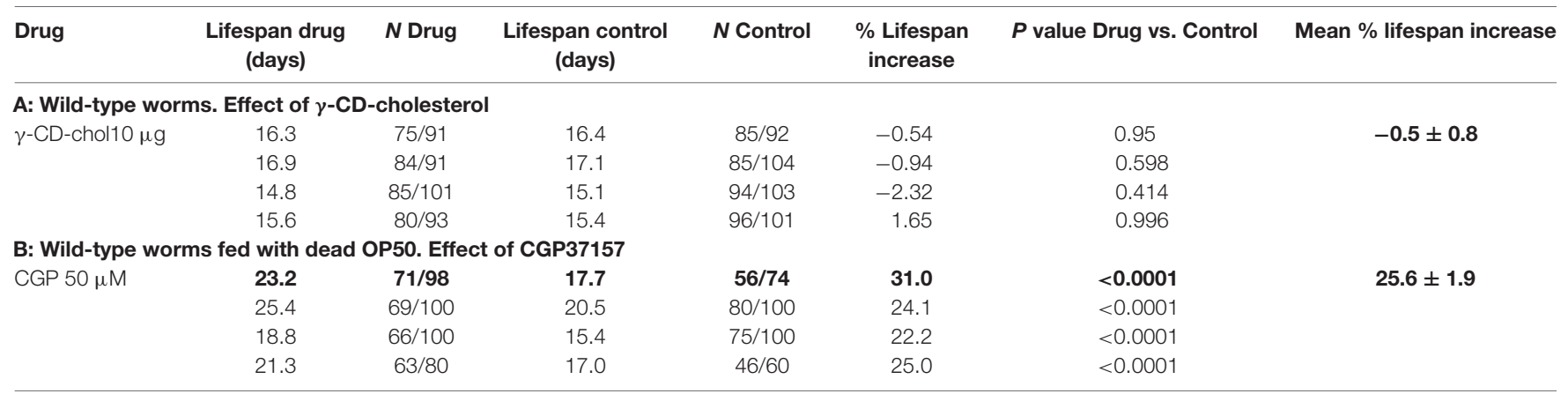

Control lifespan assays. Part A: effect of the drug carrier $\gamma$-CD, studied using a $\gamma$-CD-cholesterol inclusion compound. Part B: effect of $50 \mu M$ CGP37157 in wild-type worms fed with dead OP50. The table shows a series of lifespan assays performed in the presence or in the absence of either $\gamma$-CD-cholesterol (A) or CGP37157 (B). Other details as in Table 1.

\begin{tabular}{|c|c|c|c|c|c|c|c|}
\hline Drug & $\begin{array}{l}\text { Lifespan drug } \\
\text { (days) }\end{array}$ & $N$ Drug & $\begin{array}{c}\text { Lifespan } \\
\text { control (days) }\end{array}$ & N Control & $\begin{array}{l}\% \text { Lifespan } \\
\text { increase }\end{array}$ & $P$ value Drug vs. Control & Mean $\%$ lifespan increase \\
\hline \multirow[t]{3}{*}{ CGP $1 \mu \mathrm{g}$} & 30.4 & $72 / 93$ & 25.9 & $89 / 107$ & 17.6 & $<0.0001$ & $13.0 \pm 2.7$ \\
\hline & 26.8 & 67/101 & 24.7 & $88 / 121$ & 8.3 & $<0.0001$ & \\
\hline & 26.1 & $110 / 123$ & 23.1 & $79 / 84$ & 13.1 & $<0.0001$ & \\
\hline CGP $3 \mu \mathrm{g}$ & 26.6 & $57 / 97$ & 25.9 & $89 / 107$ & 2.9 & 0.696 & $1.4 \pm 0.9$ \\
\hline \multicolumn{8}{|l|}{ nuo-6 } \\
\hline \multirow[t]{3}{*}{ CGP $1 \mu \mathrm{g}$} & 34.8 & $117 / 140$ & 32.7 & $115 / 151$ & 6.3 & $<0.021$ & $-1.2 \pm 3.8$ \\
\hline & 30.0 & $101 / 141$ & 31.0 & $84 / 115$ & -3.3 & 0.166 & \\
\hline & 33.1 & $52 / 100$ & 35.4 & $27 / 55$ & -6.6 & 0.197 & \\
\hline \multicolumn{8}{|c|}{ daf-15/unc-24 } \\
\hline CGP $50 \mu \mathrm{M}$ & 19.0 & $82 / 101$ & 18.2 & $81 / 100$ & 4.0 & 0.069 & $-0.2 \pm 3.0$ \\
\hline \multicolumn{8}{|l|}{ unc-24/+ } \\
\hline \multirow{2}{*}{ CGP $50 \mu \mathrm{M}$} & 18.7 & 89/99 & 15.7 & $81 / 97$ & 18.9 & $<0.0001$ & \\
\hline & 19.0 & $125 / 140$ & 15.8 & $105 / 126$ & 19.8 & $<0.0001$ & \\
\hline \multicolumn{8}{|l|}{ daf-2 } \\
\hline \multirow[t]{2}{*}{ CGP $50 \mu \mathrm{M}$} & 31.2 & $62 / 101$ & 29.4 & $67 / 91$ & 6.1 & $<0.024$ & $3.3 \pm 2.9$ \\
\hline & 25.8 & $92 / 148$ & 25.7 & $75 / 146$ & 0.4 & 0.628 & \\
\hline
\end{tabular}

Lifespan assays performed with CGP37157 in several mutant C. elegans strains. The table shows a series of lifespan assays performed in several C. elegans mutant worms in the

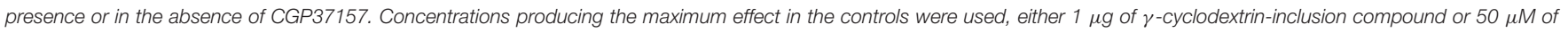
the compound dissolved in NGM agar. In bold, series shown in the survival plots of Figure 2. Other details as in Table 1.

on some of its known targets. Regarding the assays performed with 50-150 $\mu \mathrm{M}$ CGP37157 dissolved in NGM agar, we have to consider that the drug concentrations in NGM agar generally required to produce effects in C. elegans are 10-100 times higher than in cell cultures.

We have also studied the possible effect of CGP37157 on C. elegans fertility by counting the number of eggs laid per worm every $24 \mathrm{~h}$, either in the presence or in the absence of $50 \mu \mathrm{M}$ CGP37157. Figure 3A shows that the compound had no significant effect on the total number of eggs laid. We could observe, however, that laying of eggs took place with a small delay in the worms treated with the compound, although the difference was statistically significant only at day 2 of adult life (Figure 3B). A similar trend was observed after that in both groups.
Our results show a novel correlation between neuroprotective activity in cell cultures and lifespan extension in the C. elegans model for the benzothiazepine CGP37157, both effects requiring functional mitochondria to develop. Another example of this correlation is the neuroprotection and lifespan extension induced by partial pharmacological uncoupling of mitochondrial oxidative phosphorylation. Mitochondrial depolarization has been proposed to be a potential therapeutic strategy for several human disorders that involve metabolic and mitochondrial oxidative stress, including Parkinson's and Alzheimer's diseases (Wu et al., 2011; Geisler et al., 2017), cerebral ischemia (Korde et al., 2005) and heart ischemia (Brennan et al., 2006). At the same time, partial mitochondrial uncoupling attenuates age-dependent neurodegeneration and increases survival in C. elegans (Lemire et al., 2009; Cho et al., 


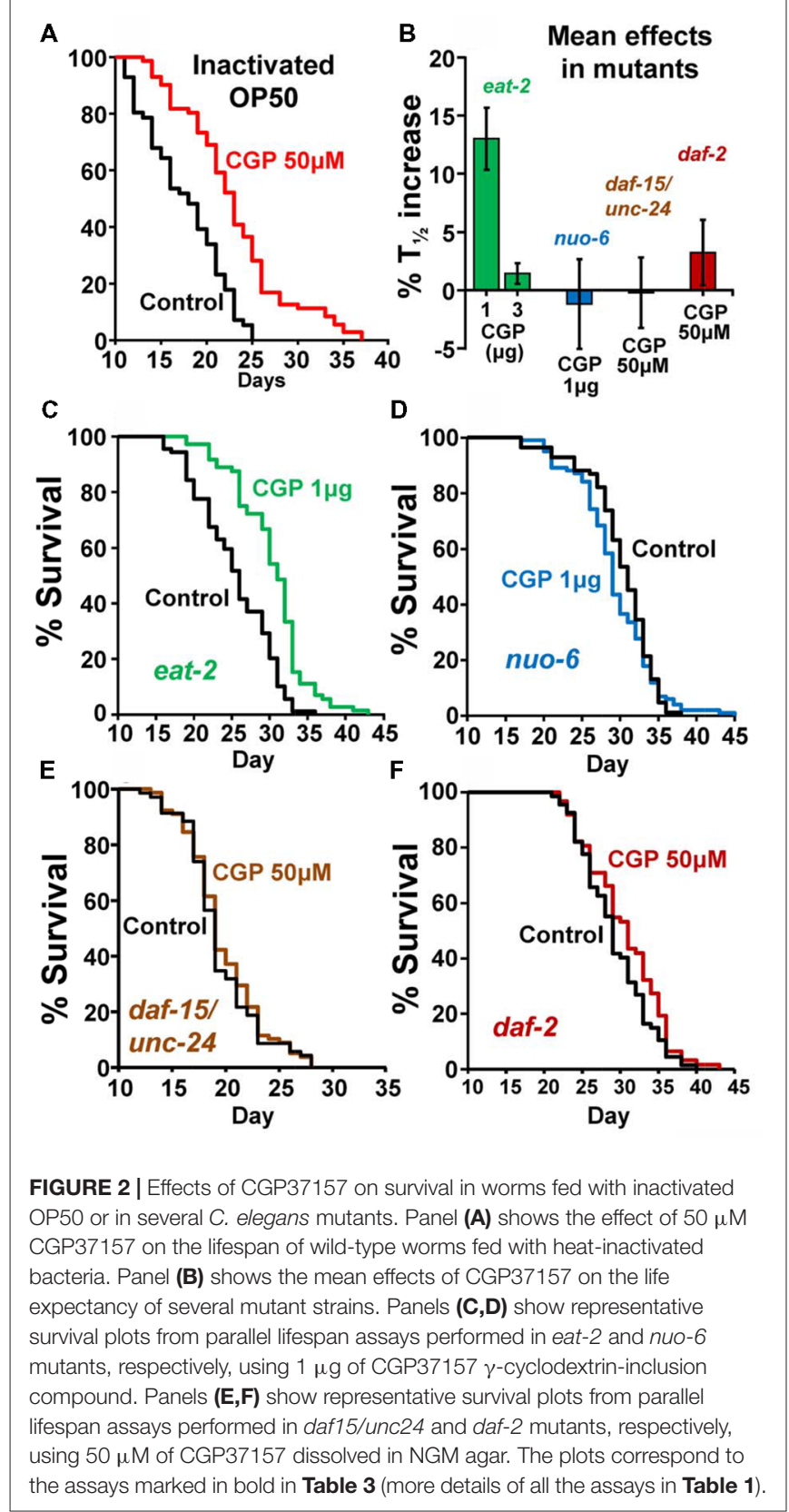

2017). Thus, mild mitochondrial dysfunction increases lifespan and triggers neuroprotection, perhaps through a hormetic response (Haigis and Yankner, 2010; López-Otín et al., 2013).

The role of mitochondrial dysfunction in aging is complex. There is a growing body of evidence suggesting that impaired mitochondrial function may protect against aging and age-associated diseases. In C. elegans, a large number of respiratory chain loss of function mutants have been studied, and many of them show higher lifespan (Yang and Hekimi, 2010b; Munkácsy and Rea, 2014). The reason for this paradoxical effect is still under debate (Dancy et al., 2014; Maglioni et al., 2014; Munkácsy and Rea, 2014; Hekimi et al., 2016). In the
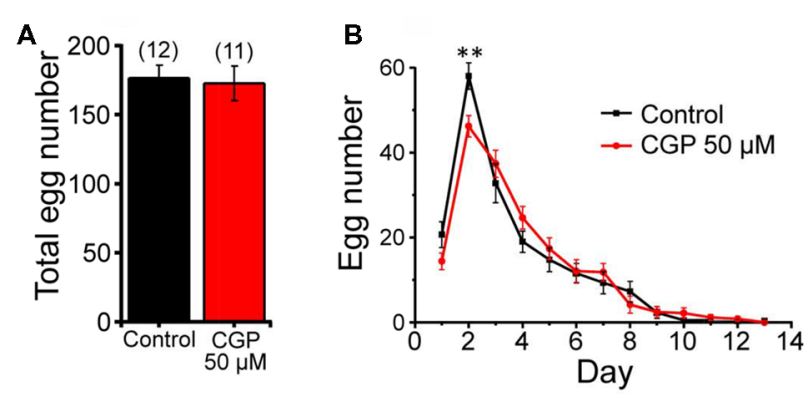

FIGURE 3 | Effects of CGP37157 on egg laying. Panel (A) shows the total number of eggs laid per worm (mean $\pm \mathrm{SE}$ ) in the absence or in the presence of $50 \mu \mathrm{M}$ CGP37157. Panel (B) shows the distribution of eggs laid per day of adult life (mean $\pm \mathrm{SE}$ ). The differences were not significant, except for day 2 $(* * p<0.01)$.

case of nuo-6 mutants, it was found that a mild elevation of mitochondrial $\mathrm{O}_{2}^{-}$was both necessary and sufficient for the increase in life expectancy (Yang and Hekimi, 2010a; Schaar et al., 2015). The idea is that moderate elevations in ROS may trigger compensatory responses which finally lead to prolonged lifespan. The fact that CGP37157 does not increase life expectancy in nuo-6 mitochondrial respiratory chain mutants suggests that lifespan extension by this compound may use a similar pathway. A possible mechanism could involve partial mitochondrial $\mathrm{Na}^{+} / \mathrm{Ca}^{2+}$ exchanger inhibition, leading to mitochondrial $\mathrm{Ca}^{2+}$ accumulation and increased $\mathrm{O}_{2}^{-}$production. Elevated ROS have also been shown to be very important for the increase in lifespan in daf-2 mutants (Zarse et al., 2012; Senchuk et al., 2018) and they modulate TOR signaling (Schieber and Chandel, 2014), This could explain why CGP37157 is not effective in mutants of these pathways, but further work will be necessary to clarify the molecular mechanism of its effect.

\section{DATA AVAILABILITY}

The raw data supporting the conclusions of this manuscript will be made available by the authors, without undue reservation, to any qualified researcher.

\section{AUTHOR CONTRIBUTIONS}

MM and JA designed the project. PG-C performed most of the lifespan experiments, and JA-V and PA-I joined in performing some of them. CR and AW made the synthesis and HPLCMS/MS measurements of CGP37157. JA wrote the manuscript. $\mathrm{RF}, \mathrm{MM}, \mathrm{CR}$, and AW helped in discussing and editing the manuscript. All authors read and approved the final manuscript.

\section{FUNDING}

This work was supported by grants from the Spanish Ministry of Economy, Industry and Competitiveness, Government of Spain (Ministerio de Economía, Industria y Competitividad, 
Gobierno de España) to MM and JA (BFU2014-55731-R and BFU2017-83509-R), projects co-financed by the European Union through the European Regional Development Fund, a grant from the Ministry of Education, Board of Castilla y León (Consejería de Educación, Junta de Castilla y León; VA011G18) to JA and a grant from Instituto de Salud Carlos III to CR (Proyectos de Investigación en Salud: PI16/01041, co-financed

\section{REFERENCES}

Alvarez-Illera, P., Sanchez-Blanco, A., Lopez-Burillo, S., Fonteriz, R. I., Alvarez, J., and Montero, M. (2016). Long-term monitoring of $\mathrm{Ca}^{2+}$ dynamics in C. elegans pharynx: an in vivo energy balance sensor. Oncotarget 7 , 67732-67747. doi: 10.18632/oncotarget.12177

Baron, K. T., and Thayer, S. A. (1997). CGP37157 modulates mitochondrial $\mathrm{Ca}^{2+}$ homeostasis in cultured rat dorsal root ganglion neurons. Eur. J. Pharmacol. 340, 295-300. doi: 10.1016/s0014-2999(97)01433-7

Brennan, J. P., Southworth, R., Medina, R. A., Davidson, S. M., Duchen, M. R., and Shattock, M. J. (2006). Mitochondrial uncoupling, with low concentration FCCP, induces ROS-dependent cardioprotection independent of KATP channel activation. Cardiovasc. Res. 72, 313-321. doi: 10.1016/j.cardiores.2006. 07.019

Buendia, I., Tenti, G., Michalska, P., Méndez-López, I., Luengo, E., Satriani, M., et al. (2017). ITH14001, a CGP37157-nimodipine hybrid designed to regulate calcium homeostasis and oxidative stress, exerts neuroprotection in cerebral ischemia. ACS Chem. Neurosci. 8, 67-81. doi: 10.1021/acschemneuro.6b 00181

Cho, I., Song, H.-O., and Cho, J. H. (2017). Mitochondrial uncoupling attenuates age-dependent neurodegeneration in C. elegans. Mol. Cells 40, 864-870. doi: 10.14348/molcells.2017.0172

Cooper, J. F., Dues, D. J., Spielbauer, K. K., Machiela, E., Senchuk, M. M., and Van Raamsdonk, J. M. (2015). Delaying aging is neuroprotective in Parkinson's disease: a genetic analysis in C. elegans models. NPJ Parkinsons Dis. 1:15022. doi: 10.1038/npjparkd.2015.22

Czyz, A., and Kiedrowski, L. (2003). Inhibition of plasmalemmal $\mathrm{Na}^{+} / \mathrm{Ca}^{2+}$ exchange by mitochondrial $\mathrm{Na}^{+} / \mathrm{Ca}^{2}+$ exchange inhibitor 7 -chloro-5-(2chlorophenyl)-1,5-dihydro-4,1-benzothiazepin-2(3H)-one (CGP-37157) in cerebellar granule cells. Biochem. Pharmacol. 66, 2409-2411. doi: 10.1016/j. bcp.2003.08.024

Dancy, B. M., Sedensky, M. M., and Morgan, P. G. (2014). Effects of the mitochondrial respiratory chain on longevity in C. elegans. Exp. Gerontol. 56, 245-255. doi: 10.1016/j.exger.2014.03.028

Gami, M. S., and Wolkow, C. A. (2006). Studies of Caenorhabditis elegans DAF-2/insulin signaling reveal targets for pharmacological manipulation of lifespan. Aging Cell 5, 31-37. doi: 10.1111/j.1474-9726.2006.00188.x

Gao, S., and Zhen, M. (2011). Action potentials drive body wall muscle contractions in Caenorhabditis elegans. Proc. Natl. Acad. Sci. U S A 108, 2557-2562. doi: 10.1073/pnas.1012346108

Geisler, J. G., Marosi, K., Halpern, J., and Mattson, M. P. (2017). DNP, mitochondrial uncoupling, and neuroprotection: a little dab'll do ya. Alzheimers Dement. 13, 582-591. doi: 10.1016/j.jalz.2016.08.001

González-Lafuente, L., Egea, J., León, R., Martínez-Sanz, F. J., Monjas, L., Perez, C., et al. (2012). Benzothiazepine CGP37157 and its isosteric 2'-ethyl analogue provide neuroprotection and block cell calcium entry. ACS Chem. Neurosci. 3, 519-529. doi: 10.1021/cn300009e

Görlach, A., Bertram, K., Hudecova, S., and Krizanova, O. (2015). Calcium and ROS: a mutual interplay. Redox Biol. 6, 260-271. doi: 10.1016/j.redox.2015. 08.010

Haigis, M. C., and Yankner, B. A. (2010). The aging stress response. Mol. Cell 40, 333-344. doi: 10.1016/j.molcel.2010.10.002

Hansen, M., Taubert, S., Crawford, D., Libina, N., Lee, S.-J., and Kenyon, C. (2007). Lifespan extension by conditions that inhibit translation in Caenorhabditis elegans. Aging Cell 6, 95-110. doi: 10.1111/j.1474-9726.2006.00267.x

$\mathrm{He}$, C., and O'Halloran, D. M. (2014). Analysis of the $\mathrm{Na}^{+} / \mathrm{Ca}^{2+}$ exchanger gene family within the phylum Nematoda. PLoS One 9:e112841. doi: 10.1371/journal.pone.0112841 by FEDER). Some C. elegans strains were provided by the Caenorhabditis Genetics Center (CGC), which is funded by NIH Office of Research Infrastructure Programs (P40 OD010440). JA-V has a fellowship from Junta de Castilla y León (JCyL), co-financed by the Fondo Social Europeo (FSE). PG-C has a FPI fellowship from Ministry of Economy, Industry and Competitiveness.

Hekimi, S., Wang, Y., and Noë, A. (2016). Mitochondrial ROS and the effectors of the intrinsic apoptotic pathway in aging cells: the discerning killers!. Front. Genet. 7:161. doi: 10.3389/fgene.2016.00161

Hernández-SanMiguel, E., Vay, L., Santo-Domingo, J., Lobatón, C. D., Moreno, A., Montero, M., et al. (2006). The mitochondrial $\mathrm{Na}^{+} / \mathrm{Ca}^{2+}$ exchanger plays a key role in the control of cytosolic $\mathrm{Ca}^{2+}$ oscillations. Cell Calcium 40, 53-61. doi: 10.1016/j.ceca.2006.03.009

Jia, K., Chen, D., and Riddle, D. (2004). The TOR pathway interacts with the insulin signaling pathway to regulate C. elegans larval development, metabolism and life span. Development 131, 3897-3906. doi: 10.1242/dev.01255

Jospin, M., Jacquemond, V., Mariol, M. C., Ségalat, L., and Allard, B. (2002). The L-type voltage-dependent $\mathrm{Ca}^{2+}$ channel EGL-19 controls body wall muscle function in Caenorhabditis elegans. J. Cell Biol. 159, 337-347. doi: 10.1083/jcb. 200203055

Kashima, N., Fujikura, Y., Komura, T., Fujiwara, S., Sakamoto, M., Terao, K., et al. (2012). Development of a method for oral administration of hydrophobic substances to Caenorhabditis elegans: pro-longevity effects of oral supplementation with lipid-soluble antioxidants. Biogerontology 13, 337-344. doi: 10.1007/s10522-012-9378-3

Korde, A. S., Pettigrew, L. C., Craddock, S. D., and Maragos, W. F. (2005). The mitochondrial uncoupler 2,4-dinitrophenol attenuates tissue damage and improves mitochondrial homeostasis following transient focal cerebral ischemia. J. Neurochem. 94, 1676-1684. doi: 10.1111/j.1471-4159.2005. 03328.x

Lakowski, B., and Hekimi, S. (1998). The genetics of caloric restriction in Caenorhabditis elegans. Proc. Natl. Acad. Sci. U S A 95, 13091-13096. doi: 10.1073/pnas.95.22.13091

Lapierre, L. R., and Hansen, M. (2012). Lessons from C. elegans: signaling pathways for longevity. Trends Endocrinol. Metab. 23, 637-644. doi: 10.1016/j.tem.2012. 07.007

Lee, R. Y., Lobel, L., Hengartner, M., Horvitz, H. R., and Avery, L. (1997). Mutations in the alphal subunit of an L-type voltage-activated $\mathrm{Ca}^{2+}$ channel cause myotonia in Caenorhabditis elegans. ЕMBO J. 16, 6066-6076. doi: 10.1093/emboj/16.20.6066

Lemire, B. D., Behrendt, M., DeCorby, A., and Gášková, D. (2009). C. elegans longevity pathways converge to decrease mitochondrial membrane potential. Mech. Ageing Dev. 130, 461-465. doi: 10.1016/j.mad.2009.05.001

Liu, P., Ge, Q., Chen, B., Salkoff, L., Kotlikoff, M. I., and Wang, Z. W. (2011). Genetic dissection of ion currents underlying all-or-none action potentials in C. elegans body-wall muscle cells. J. Physiol. 589, 101-117. doi: 10.1113/jphysiol.2010.200683

López-Otín, C., Blasco, M. A., Partridge, L., Serrano, M., and Kroemer, G. (2013). The hallmarks of aging. Cell 153, 1194-1217. doi: 10.1016/j.cell.2013.05.039

Maglioni, S., Schiavi, A., Runci, A., Shaik, A., and Ventura, N. (2014). Mitochondrial stress extends lifespan in C. elegans through neuronal hormesis. Exp. Gerontol. 56, 89-98. doi: 10.1016/j.exger.2014.03.026

Martínez-Sanz, F. J., Lajarín-Cuesta, R., González-Lafuente, L., MorenoOrtega, A. J., Punzón, E., Cano-Abad, M. F., et al. (2016). Neuroprotective profile of pyridothiazepines with blocking activity of the mitochondrial $\mathrm{Na}^{+} / \mathrm{Ca}^{2+}$ exchanger. Eur. J. Med. Chem. 109, 114-123. doi: 10.1016/j.ejmech. 2015.12.043

Martínez-Sanz, F. J., Lajarín-Cuesta, R., Moreno-Ortega, A. J., GonzálezLafuente, L., Fernández-Morales, J. C., López-Arribas, R., et al. (2015). Benzothiazepine CGP37157 analogues exert cytoprotection in various in vitro models of neurodegeneration. ACS Chem. Neurosci. 6, 1626-1636 doi: 10.1021/acschemneuro.5b00161

Moreno-Ortega, A. J., Martínez-Sanz, F. J., Lajarín-Cuesta, R., de Los Rios, C., and Cano-Abad, M. F. (2015). Benzothiazepine CGP37157 and its 2'-isopropyl 
analogue modulate $\mathrm{Ca}^{2+}$ entry through CALHM1. Neuropharmacology 95, 503-510. doi: 10.1016/j.neuropharm.2015.02.016

Munkácsy, E., and Rea, S. L. (2014). The paradox of mitochondrial dysfunction and extended longevity. Exp. Gerontol. 56, 221-233. doi: 10.1016/j.exger.2014. 03.016

Nicolau, S. M., de Diego, A. M. G., Cortés, L., Egea, J., González, J. C., Mosquera, M., et al. (2009). Mitochondrial $\mathrm{Na}^{+} / \mathrm{Ca}^{2+}$-exchanger blocker CGP37157 protects against chromaffin cell death elicited by veratridine. J. Pharmacol. Exp. Ther. 330, 844-854. doi: 10.1124/jpet.109.154765

Nicolau, S. M., Egea, J., López, M. G., and García, A. G. (2010). Mitochondrial $\mathrm{Na}^{+} / \mathrm{Ca}^{2+}$ exchanger, a new target for neuroprotection in rat hippocampal slices. Biochem. Biophys. Res. Commun. 400, 140-144. doi: 10.1016/j.bbrc.2010. 08.028

Palty, R., Silverman, W. F., Hershfinkel, M., Caporale, T., Sensi, S. L., Parnis, J., et al. (2010). NCLX is an essential component of mitochondrial $\mathrm{Na}^{+} / \mathrm{Ca}^{2+}$ exchange. Proc. Natl. Acad. Sci. U S A 107, 436-441. doi: 10.1073/pnas. 0908099107

Pei, Y., Lilly, M. J., Owen, D. J., D’Souza, L. J., Tang, X. Q., Yu, J., et al. (2003). Efficient syntheses of benzothiazepines as antagonists for the mitochondrial sodium-calcium exchanger: potential therapeutics for type II diabetes. J. Org. Chem. 68, 92-103. doi: 10.1021/jo020446t

Ruiz, A., Alberdi, E., and Matute, C. (2014). CGP37157, an inhibitor of the mitochondrial $\mathrm{Na}^{+} / \mathrm{Ca}^{2+}$ exchanger, protects neurons from excitotoxicity by blocking voltage-gated $\mathrm{Ca}^{2+}$ channels. Cell Death Dis. 5:e1156. doi: $10.1038 /$ cddis. 2014.134

Schaar, C. E., Dues, D. J., Spielbauer, K. K., Machiela, E., Cooper, J. F., Senchuk, M., et al. (2015). Mitochondrial and cytoplasmic ROS have opposing effects on lifespan. PLoS Genet. 11:e1004972. doi: 10.1371/journal.pgen.1004972

Schieber, M., and Chandel, N. S. (2014). TOR signaling couples oxygen sensing to lifespan in C. elegans. Cell Rep. 9, 9-15. doi: 10.1016/j.celrep.2014.08.075

Senchuk, M. M., Dues, D. J., Schaar, C. E., Johnson, B. K., Madaj, Z. B., Bowman, M. J., et al. (2018). Activation of DAF-16/FOXO by reactive oxygen species contributes to longevity in long-lived mitochondrial mutants in Caenorhabditis elegans. PLoS Genet. 14:e1007268. doi: 10.1371/journal.pgen. 1007268

Sharma, V., He, C., Sacca-Schaeffer, J., Brzozowski, E., Martin-Herranz, D. E., Mendelowitz, Z., et al. (2013). Insight into the family of $\mathrm{Na}^{+} / \mathrm{Ca}^{2+}$ exchangers of Caenorhabditis elegans. Genetics 195, 611-619. doi: 10.1534/genetics.113. 153106

Sharma, V., and O'Halloran, D. M. (2014). Recent structural and functional insights into the family of sodium calcium exchangers. Genesis 52, 93-109. doi: $10.1002 /$ dvg. 22735

Sharma, V., Roy, S., Sekler, I., and O'Halloran, D. M. (2017). The NCLX-type $\mathrm{Na}^{+} / \mathrm{Ca}^{2+}$ exchanger NCX-9 is required for patterning of neural circuits in Caenorhabditis elegans. J. Biol. Chem. 292, 5364-5377. doi: 10.1074/jbc.M116. 758953
Shtonda, B., and Avery, L. (2005). CCA-1, EGL-19 and EXP-2 currents shape action potentials in the Caenorhabditis elegans pharynx. J. Exp. Biol. 208, 2177-2190. doi: 10.1242/jeb.01615

So, S., Miyahara, K., and Ohshima, Y. (2011). Control of body size in C. elegans dependent on food and insulin/IGF-1 signal. Genes Cells 16, 639-651. doi: $10.1111 / j .1365-2443.2011 .01514 . x$

Stiernagle, T. (2006). "Maintenance of C. elegans," in WormBook, ed. The C. elegans Research Community (Minneapolis, MN: WormBook), 1-11. doi: 10.1895/wormbook.1.101.1

Tanis, J. E., Ma, Z., Krajacic, P., He, L., Foskett, J. K., and Lamitina, T. (2013). CLHM-1 is a functionally conserved and conditionally toxic $\mathrm{Ca}^{2+}$. permeable ion channel in Caenorhabditis elegans. J. Neurosci. 33, 12275-12286. doi: 10.1523/JNEUROSCI.5919-12.2013

Wu, Y. N., Munhall, A. C., and Johnson, S. W. (2011). Mitochondrial uncoupling agents antagonize rotenone actions in rat substantia nigra dopamine neurons. Brain Res. 1395, 86-93. doi: 10.1016/j.brainres.2011. 04.032

Yang, W., and Hekimi, S. (2010a). A mitochondrial superoxide signal triggers increased longevity in Caenorhabditis elegans. PLoS Biol. 8:e1000556. doi: 10.1371 /journal.pbio.1000556

Yang, W., and Hekimi, S. (2010b). Two modes of mitochondrial dysfunction lead independently to lifespan extension in Caenorhabditis elegans. Aging Cell 9, 433-447. doi: 10.1111/j.1474-9726.2010.00571.x

Yee, C., Yang, W., and Hekimi, S. (2014). The intrinsic apoptosis pathway mediates the pro-longevity response to mitochondrial ROS in C. elegans. Cell 157, 897-909. doi: 10.1016/j.cell.2014.02.055

Zárate, S., Stevnsner, T., and Gredilla, R. (2017). Role of estrogen and other sex hormones in brain aging. Neuroprotection and DNA repair. Front. Aging Neurosci. 9:430. doi: 10.3389/fnagi.2017.00430

Zarse, K., Schmeisser, S., Groth, M., Priebe, S., Beuster, G., Kuhlow, D., et al. (2012). Impaired insulin/IGF1 signaling extends life span by promoting mitochondrial L-proline catabolism to induce a transient ROS signal. Cell Metab. 15, 451-465. doi: 10.1016/j.cmet.2012.02.013

Conflict of Interest Statement: The authors declare that the research was conducted in the absence of any commercial or financial relationships that could be construed as a potential conflict of interest.

Copyright (ㄷ 2019 García-Casas, Arias-del-Val, Alvarez-Illera, Wojnicz, de los Ríos, Fonteriz, Montero and Alvarez. This is an open-access article distributed under the terms of the Creative Commons Attribution License (CC BY). The use, distribution or reproduction in other forums is permitted, provided the original author(s) and the copyright owner(s) are credited and that the original publication in this journal is cited, in accordance with accepted academic practice. No use, distribution or reproduction is permitted which does not comply with these terms. 